\title{
Down-regulation of Connexin43 in Early Myocardial Ischemia and Protective Effect by Ischemic Preconditioning in Rat Hearts In Vivo
}

\author{
Kazuhito HatanAKA, ${ }^{1} \mathrm{MD}$, Hiroyuki KawATA, ${ }^{2} \mathrm{MD}$, \\ Toshihiko ToyofuKu, ${ }^{3} \mathrm{MD}$, and Ken-ichi YoshIDA, ${ }^{1} \mathrm{MD}$
}

\begin{abstract}
SUMMARY
Connexin $43(\mathrm{Cx} 43)$, a primary component of gap junctions, contributes to intercellular electrochemical communication. $\mathrm{Cx} 43$ undergoes dephosphorylation in early ischemia. We examined whether $\mathrm{Cx} 43$ is degraded in association with dephosphorylation during early myocardial ischemia and whether ischemic preconditioning (IP) affects the degradation after rat coronary artery occlusion. Male Sprague-Dawley rats underwent coronary artery occlusion for 1,2 , or 3 hours, or for 1 hour following treatment either with a calcineurin inhibitor (cyclosporine A), proteasome inhibitor (PSI), or lysosomal inhibitor (E64c), or following IP alone or after protein kinase C (PKC) inhibitor (chelerythrine) pretreatment. The IP was afforded by three cycles of 3 minute ischemia and 5 minute reperfusion. A large portion of the phosphorylated $\mathrm{Cx} 43(\mathrm{pCx} 43)$ in the membrane fraction was dephosphorylated, while a small portion was degraded at 1 hour of ischemia. The effects of the inhibitors were dephosphorylation and degradation by calcineurin and proteasome/lysosome, respectively. IP suppressed the decrease in $\mathrm{pCx} 43$ and increase in $\mathrm{dCx} 43$, while only the former was inhibited by the PKC inhibitor chelerythrine. The Cx43 mRNA level was reduced at 3 hours, but not at 1 hour of ischemia, irrespective of IP. We believe that $\mathrm{Cx} 43$ is dephosphorylated and degraded in early ischemia, whereas Cx43 transcription was suppressed at a later phase of ischemia. (Jpn Heart J 2004; 45: 1007-
\end{abstract} 1019)

Key words: Connexin 43, Myocardial ischemia, Dephosphorylation, Degradation, Ischemic preconditioning, Protein kinase $\mathrm{C}$

Cardiac gap junctions (GJ), composed mainly of connexin43 (Cx43), regulate electrochemical coupling between cardiomyocytes, thereby coordinating the contraction. ${ }^{1)}$ Gap junctional properties can be regulated by protein kinase $\mathrm{C}$ (PKC), mitogen-activated protein kinase, and src protein kinase, among others. ${ }^{1)}$

From the ${ }^{1}$ Department of Forensic Medicine, Graduate School of Medicine, University of Tokyo, Tokyo, ${ }^{2}$ The $\quad$ First Department of Internal Medicine, Nara Medical University, Nara, and ${ }^{3}$ Departments of Internal Medicine and Therapeutics, and Pathophysiology, Osaka University Graduate School of Medicine, Osaka, Japan.

Address for correspondence: Ken-ichi Yoshida, MD, Department of Forensic Medicine, Graduate School of Medicine, University of Tokyo, 7-3-1 Hongo, Bunkyo-ku, Tokyo 113-0033, Japan.

Received for publication February 16, 2004.

Revised and accepted June 3, 2004. 
Cx43 immunoreactivity lies primarily in the intercalated discs (ID) and its intensity is reduced during myocardial ischemia, ${ }^{2,3)}$ which may implicate it arrhythmogenesis. ${ }^{4)}$ In $\mathrm{Cx} 43$-deficient mice, ischemia promotes ventricular arrhythmias. ${ }^{5)}$ It has been also demonstrated that dephosphorylation and translocation of $\mathrm{Cx} 43$ from the GJ is associated with electrical uncoupling induced by ischemia. ${ }^{6)}$ On the basis of the findings that hypoxia induces $\mathrm{Cx} 43$ dephosphorylation through calcineurin and reduces $\mathrm{Cx} 43$ immunostaining in the astrocytes, it has been proposed that "epitope masking" due to dephosphorylation reduces immunostaining. ${ }^{7)}$ This hypothesis has not been evaluated in myocardial ischemia.

In normoxic perfused hearts and cultured cardiomyocytes, $\mathrm{Cx} 43$ turns over rapidly with a half-life of about 1.3 hours through proteasome and/or lysosomal proteases. ${ }^{1,8)}$ This rapid Cx43 turnover is expected to regulate GJ assembly and conductance under physiological and pathological conditions. ${ }^{1)}$ However, little is known about the role of these proteases in $\mathrm{Cx} 43$ turnover in in vivo myocardial ischemia.

Ischemic preconditioning (IP), conferred by brief ischemia-reperfusion cycles, attenuates arrhythmias, myocardial injury, left ventricular remodeling, and infarct development after subsequent prolonged ischemia (-reperfusion)..$^{9-11)}$ It is known that PKC and ATP-sensitive $\mathrm{K}^{+}$channels are involved in the protection afforded by IP. ${ }^{10,12)}$ It is reported that $\mathrm{Cx} 43$ is involved in the infarct size limitation afforded by IP as demonstrated by the comparison of heterozygous knockout and wild type mice. ${ }^{13)}$ Recently, it has been proposed that the protective effect of IP against myocardial infarction is exerted at the GJ through Cx43 modulation in ischemic myocardium. ${ }^{14-16)}$

In an in vivo model of early myocardial ischemia in rats, we addressed 1) whether $\mathrm{Cx} 43$ is degraded in association with dephosphorylation, or reduced immunostaining, 2) whether IP affects these changes, and 3) whether the transcription is modulated.

\section{Methods}

Experimental animals and protocols: In accordance with the Guidelines of the Committee on Animal Experiments of the Graduate School of Medicine, University of Tokyo, male Sprague-Dawley rats, aged 8 weeks, were anesthetized with sodium pentobarbital $(50 \mathrm{mg} / \mathrm{kg}$, i.p. $)$ and ventilated with room air with the body temperature maintained at $37-38^{\circ} \mathrm{C}$, as previously reported. ${ }^{3,10)}$ After left thoracotomy, the left anterior descending coronary artery (LAD) was occluded. The rats were randomly assigned to one of the following groups ( $n=5$ for each). In the time course study, the rats underwent LAD occlusion for 1, 2, or 3 hours. In the 
inhibitor study, the rats were pretreated either with the calcineurin inhibitor cyclosporine A (CsA) (Wako Pure Chemicals, Osaka, Japan) $(5 \mathrm{mg} / \mathrm{kg}$, i.v.) for 15 minutes, the lysosomal inhibitor E64c (Peptide Institute, Osaka, Japan) (10 $\mathrm{mg} / \mathrm{kg}$, i.p.) for 1 hour, or the proteasome inhibitor PSI (Peptide Institute) (2 mg/ $\mathrm{kg}$, i.p.) for 1 hour before LAD occlusion. In the IP study, the rats were subjected to an IP procedure consisting of 3 cycles of 3 minutes of ischemia and 5 minutes of reperfusion ${ }^{10)}$ before ischemia for 1 or 3 hours. To another group, the PKC inhibitor chelerythrine chloride (Sigma, St. Louis, MO, USA) (5 mg/kg, i.v.) was administered immediately before the IP procedure. CsA, E64c, PSI, and chelerythrine were dissolved in dimethyl-sulfoxide (DMSO) and diluted with physiological saline at final DMSO concentrations of $2 \% \mathrm{v} / \mathrm{v}$ before injection. We determined the amount and the route of the inhibitors on the basis of previous results. ${ }^{10,17-19)}$ The sham group rats underwent the same procedure without LAD occlusion, while the experimental periods and the vehicle volume were matched. At the end of the LAD occlusion, following the injection of $0.3 \%$ Evans blue solution, the hearts were quickly removed and rinsed with physiological saline. The ischemic area, demarcated from the dye-stained nonischemic area, ${ }^{10)}$ was carefully dissected and frozen at $-80^{\circ} \mathrm{C}$ until the analyses.

Biochemical analyses: As previously described ${ }^{20)}$ the frozen hearts underwent subcellular fractionation. The $1,000 \times \mathrm{g}$ pellet, $100,000 \times \mathrm{g}$ pellet, and $100,000 \times$ $\mathrm{g}$ supernatant were referred to as the P1 (nuclear-myofibril), P2 (membrane), and $\mathrm{S}$ (cytosolic) fractions, respectively. ${ }^{20,21)}$ SDS-polyacrylamide gel $(12.5 \%)$ electrophoresis and immunoblotting were performed as previously described, ${ }^{6}{ }^{6}$ with equal protein (10 $\mu \mathrm{g} / \mathrm{lane})$ loading. The PVDF membranes were incubated with the anti-Cx43 antibody (mouse monoclonal antibody or rabbit polyclonal antibody, Zymed Laboratories, South San Francisco, CA, USA) for 1 hour, and then the proteins on the membranes were visualized using an ECL Western blotting detection kit (Amersham Life Science, Buckinghamshire, UK). The band densities were quantified using an image analyzer (Densitograph AE-6920, ATTO, Tokyo). ${ }^{10)}$ For dot blotting, $25 \mu \mathrm{g}$ aliquots of the $\mathrm{S}$ fraction were spotted onto nitrocellulose membranes, which were dried and processed in a manner similar to that for the immunoblotting procedure.

Immunohistochemistry: Immunofluorescence staining was performed as previously described. ${ }^{3,6)}$ Paraformaldehyde-fixed and paraffin-embedded heart sections were treated with microwaves, blocked with 5\% BSA/TBS, incubated with the anti-Cx43 antibody overnight at $4^{\circ} \mathrm{C}$, washed with TBS, and incubated with fluorescein isothiocyanate-labeled IgG and Texas-Red (r)-labeled IgG (Vector Laboratories, Burlingame, CA, USA) for 1 hour at room temperature. The tissue sections were examined under immunofluorescence microscopy (Axioskop 2, 
Zeiss, Jena, Germany) equipped with an image analyzer (Image-Pro Plus, Media Cybernetics, Maryland, USA).

Northern blotting analysis: The Northern blotting was performed as previously described. ${ }^{22)}$ Total RNA ( $\left.2 \mu \mathrm{g}\right)$, extracted from the frozen heart, was reverse-transcribed to cDNA and amplified by polymerase chain reaction (PCR) with sequence specific primers: Cx43 sense 5'-TGGTACATCTATGGGTTCAGCTTGAGCGCG-3', and antisense 5'-CATAATCGACAGCTCGATGCTCAAGCCGGT-3'. The PCR product was recovered as a probe and labeled using a digoxigenin-11-deoxyuridine-5-triphosphatase (DIG) labeling kit (Boehringer Mannheim, Mannheim, Germany). Total RNA (20 $\mu \mathrm{g})$ was denatured, electrophoresed on $1 \%$ agarose gels, and transferred onto nylon membranes. After hybridization, the band was detected with a DIG detection kit (Boehringer Mannheim), and the density was quantified using the image analyzer and normalized to the 28S rRNA.

Statistics: The data are expressed as the mean \pm SE and were analyzed statistically by analysis of variance (ANOVA) followed by Fisher's post hoc analysis. Differences at $P<0.05$ were considered to be statistically significant.

\section{RESUlTS}

Temporal changes in $\mathrm{Cx} 43$ levels during early myocardial ischemia: The antiCx43 polyclonal antibody (pAb) reacts both with phosphorylated Cx43 (pCx43) and dephosphorylated $\mathrm{Cx} 43(\mathrm{dCx} 43)$, whereas the anti-Cx43 monoclonal antibody $(\mathrm{mAb})$ reacts selectively with $\mathrm{dCx} 43 .{ }^{23)}$ Figure 1 shows representative immunoblots of the P2 fraction, while Figure 2 shows the quantification of the

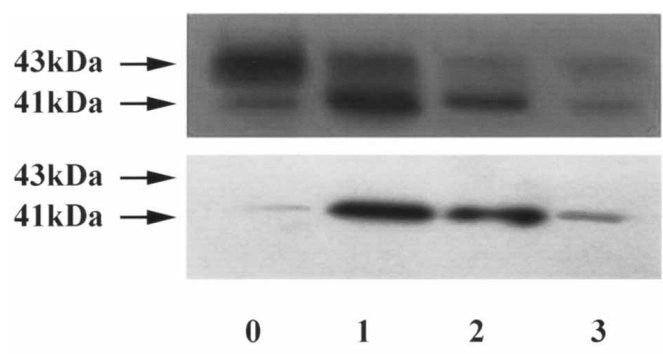

Time after LAD ligation (hours)

Figure 1. Representative immunoblots showing the temporal change in the $\mathrm{Cx} 43$ levels of the $\mathrm{P} 2$ fraction at $0,1,2$, and 3 hours of ischemia $(43 \mathrm{kDa}=$ phosphorylated $\mathrm{Cx} 43,41 \mathrm{kDa}=$ dephosphorylated $\mathrm{Cx} 43$ ). The anti-Cx43 polyclonal antibody recognizes both phosphorylated $\mathrm{Cx} 43$ and dephosphorylated $\mathrm{Cx} 43$ bands (upper blot), whereas the anti-Cx43 monoclonal antibody only recognizes dephosphorylated Cx43 bands (lower blot). 
A

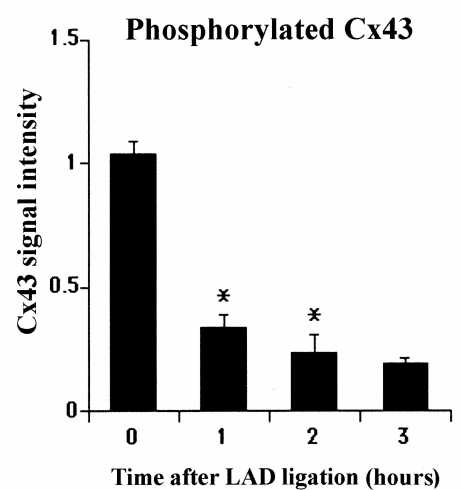

Time after LAD ligation (hours)

\section{B}

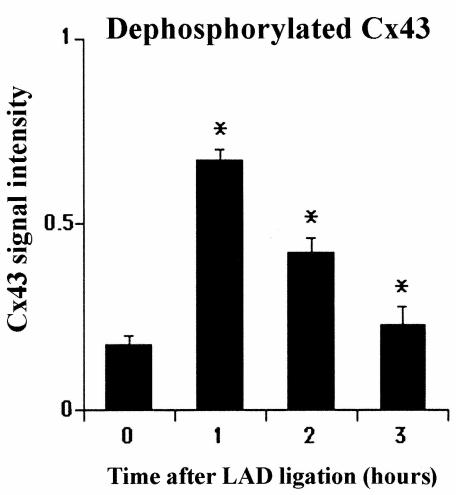

D

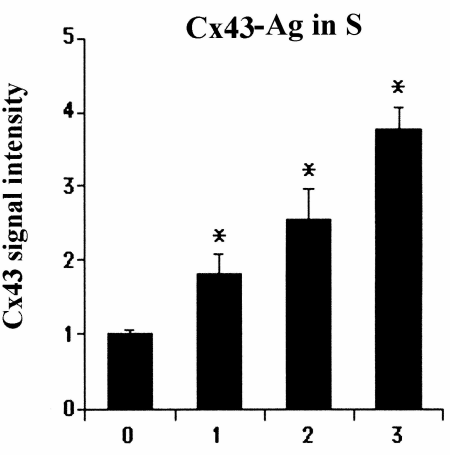

Time after LAD ligation (hours)

Figure 2. The relative densities of the $\mathrm{Cx} 43$ bands on the immunoblots are quantified as ratios relative to the mean densities before (0 hour) ischemia (A: phosphorylated $\mathrm{Cx} 43$, B: dephosphorylated $\mathrm{Cx} 43$, C: total $\mathrm{Cx} 43$ ). The significant differences between values at each 1 hour interval $(* P<0.05)$ are shown. Figure $2 \mathrm{D}$ represents the relative densities of $\mathrm{Cx} 43$ spots of the $\mathrm{S}$ fraction on dot blot quantified as ratios relative to the mean density before ( 0 hour) ischemia. The significant differences between values at each 1 hour interval $(* P<0.05)$ are shown.

Cx43 band density on the immunoblots (A, B, C) and the Cx43 dot density of the $\mathrm{S}$ fraction on the dot blots (D). The $\mathrm{P} 2$ contains the ID and sarcolemma, while the $\mathrm{S}$ contains the cytosol. ${ }^{24)}$ At 1 hour of ischemia, the $\mathrm{pCx} 43$ in the $\mathrm{P} 2$ fraction was reduced to about $30 \%$ of the levels of the sham group, whereas the $\mathrm{dCx} 43$ levels greatly increased, indicating the dephosphorylation of $\mathrm{pCx} 43$. Total Cx43 (pCx43 plus $\mathrm{dCx} 43$ ) was decreased by only about $15 \%$ at 1 hour of ischemia. The $\mathrm{pCx} 43$ levels were greatly decreased by 3 hours of ischemia, along with the reduction of $\mathrm{dCx} 43$ between 1 and 3 hours of ischemia. The $\mathrm{Cx} 43$ levels in the nonischemic area were unaltered by 3 hours of ischemia, while a $\mathrm{Cx} 43$ band was not detected 
in the P1 and $\mathrm{S}$ fractions (data not shown). Dot-blotting analysis of the $\mathrm{S}$ fraction showed an increase in $\mathrm{Cx} 43$ immunoreactivity with ischemia duration.

There was neither a reduction in triphenyltetrazolium chloride staining (infarction) nor myoglobin diffusion after 3 hours of ischemia (data not shown). By contrast, infarct size was about $24 \%$ after 3 days of ischemia, and the infarct and myoglobin diffusion were evident after approximetely 30 minutes of ischemia followed by reperfusion for more than about 3 hours in the same models (data not shown).
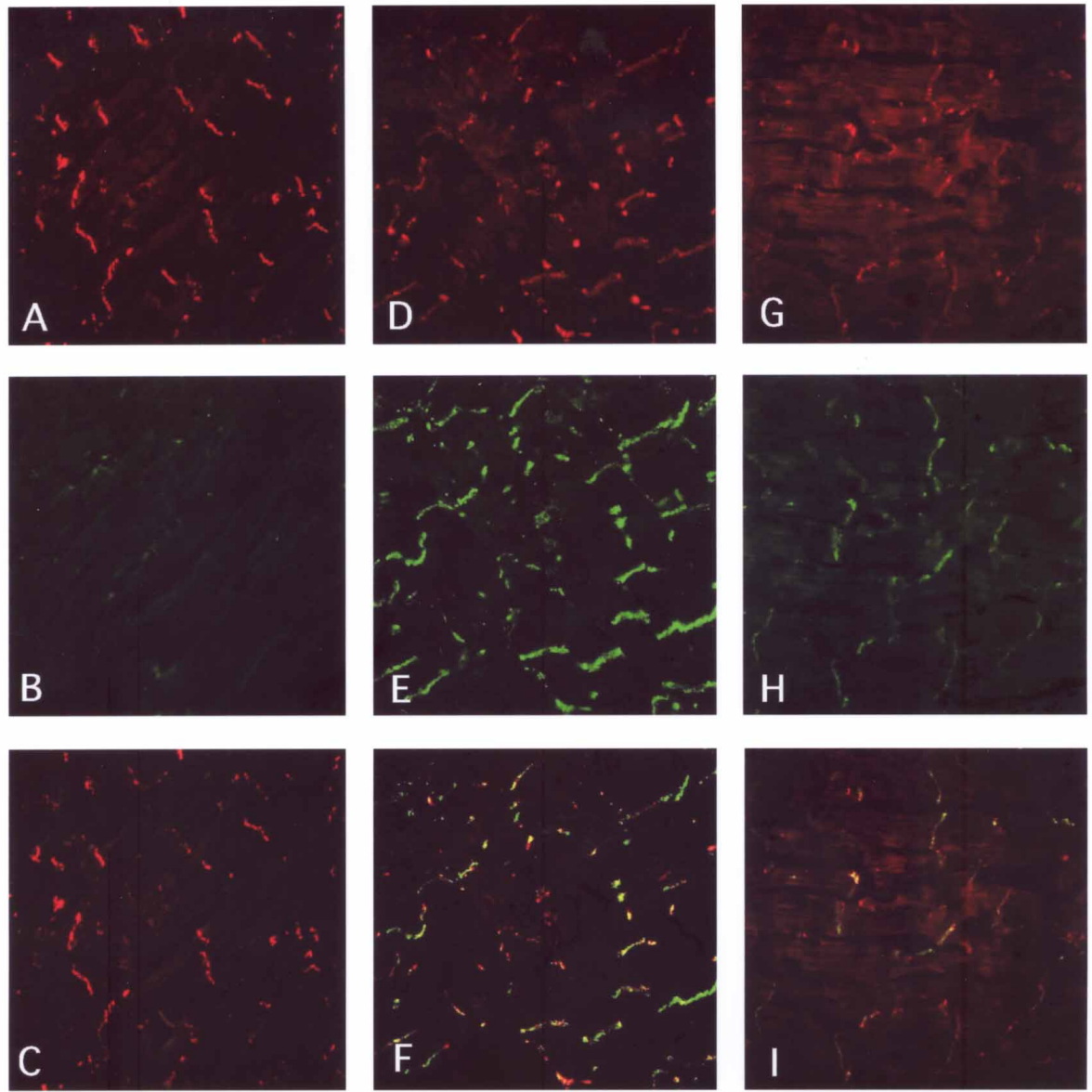

Figure 3. Immunofluorescence localization of $\mathrm{Cx} 43$ with the anti-Cx43 polyclonal antibody (A, D, G) and monoclonal antibody $(\mathbf{B}, \mathbf{E}, \mathbf{H})$ on sections from the sham $(\mathbf{A}, \mathbf{B}, \mathbf{C}), 1$ hour of ischemia $(\mathbf{D}, \mathbf{E}, \mathbf{F}), 3$ hours of ischemia $(\mathbf{G}, \mathbf{H}, \mathbf{I})$, and the corresponding fused images $(\mathbf{C}, \mathbf{F}, \mathbf{I})$. The polyclonal antibody stains the intercalated discs of the sham, whereas it stains the cytoplasm diffusely but weakly at 1 and 3 hours of ischemia. The monoclonal antibody stains the intercalated discs at 1 hour (strongly) and 3 hours of ischemia. (magnifications: $\times 400$ ). 
Figure 3 shows the immunofluorescence profiles of $\mathrm{Cx} 43$ in ischemic heart. In the sham, the total $\mathrm{Cx} 43$ levels, as detected by the $\mathrm{pAb}$, were distributed to the ID (A, B, C). At 1 hour of ischemia, the $\mathrm{dCx} 43$ signal, as detected by $\mathrm{mAb}$, was greatly increased in the ID (E, F), with a small decrease in the $\mathrm{pCx} 43$ signal (D). In the later phase of ischemia (1-3 hours), the total Cx43 signal in the ID was clearly reduced and diffusely distributed throughout the cytoplasm $(\mathrm{G}, \mathrm{H}, \mathrm{I})$. Thus, pCx43 in the ID was largely dephosphorylated during early myocardial ischemia, whereas total $\mathrm{Cx} 43$ was reduced during the later phase of ischemia, which is consistent with the biochemical analyses (Figure 2C).

Effect of cyclosporine A on Cx43 levels: We examined the effects of a calcineurin inhibitor, CsA, on the Cx43 in immunoblots after ischemia for 1 hour. CsA inhibited dephosphorylation of $\mathrm{Cx} 43$ at 1 hour of ischemia with no effect on the total Cx43 level (Figure 4).

Effects of proteasome and lysosome inhibitors on Cx43 levels: We investigated the effects of a proteasome inhibitor, PSI (Figure 5A), and a lysosomal inhibitor,
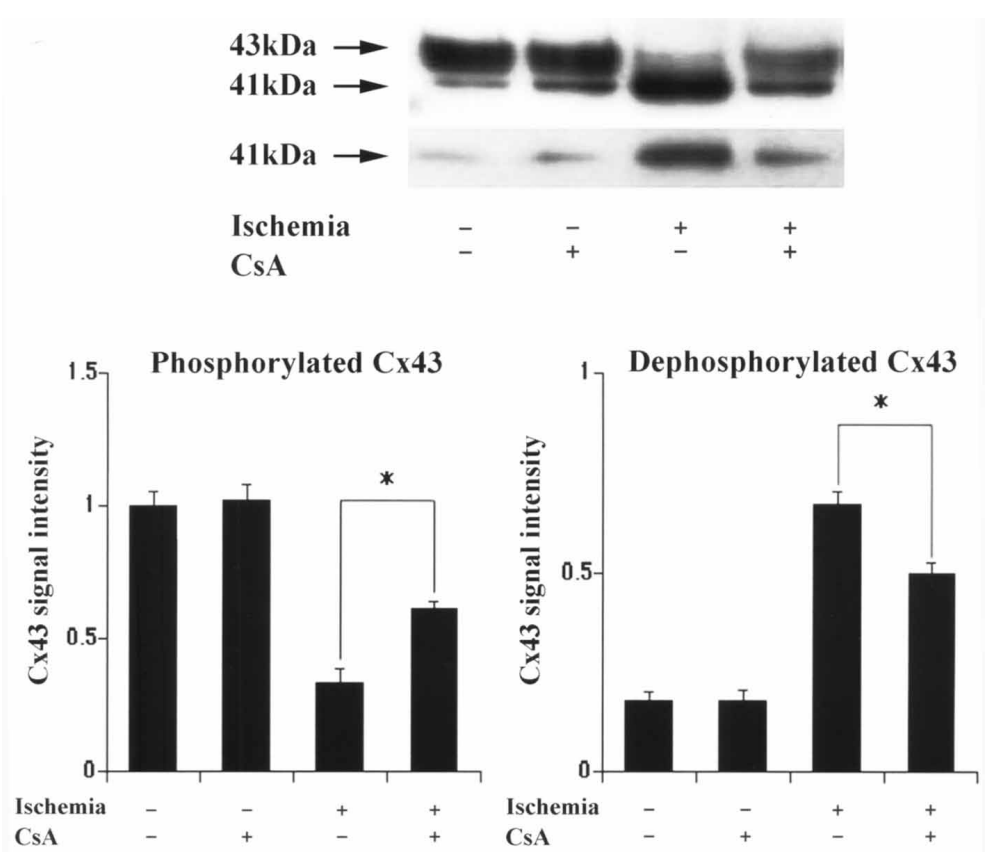

Figure 4. Representative immunoblots with the anti-Cx43 polyclonal antibody (upper) and monoclonal antibody (lower) showing the effect of CsA on phosphorylated $\mathrm{Cx} 43$ and dephosphorylated $\mathrm{Cx} 43$ levels at 1 hour of ischemia and in the sham. The relative densities of phosphorylated $\mathrm{Cx} 43$ and dephosphorylated $\mathrm{Cx} 43$ are quantified as ratios relative to the mean densities of the sham without CsA $(* P<0.05$ versus ischemia without $\mathrm{CsA})$. 
A
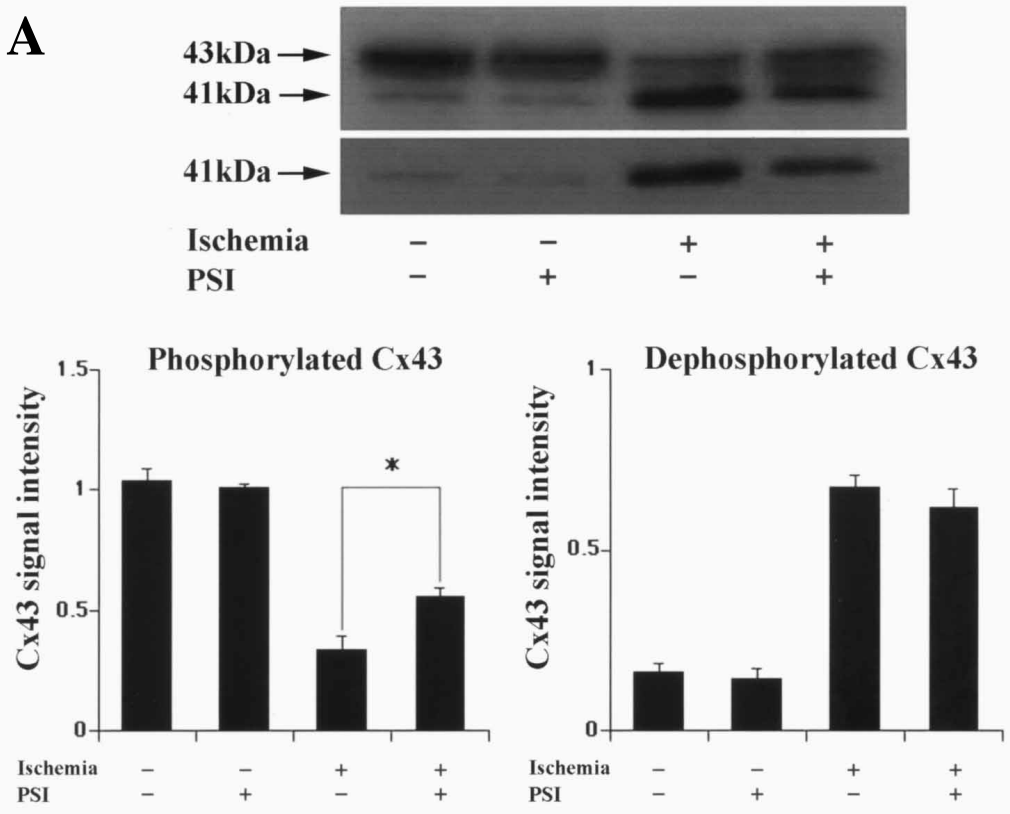

B
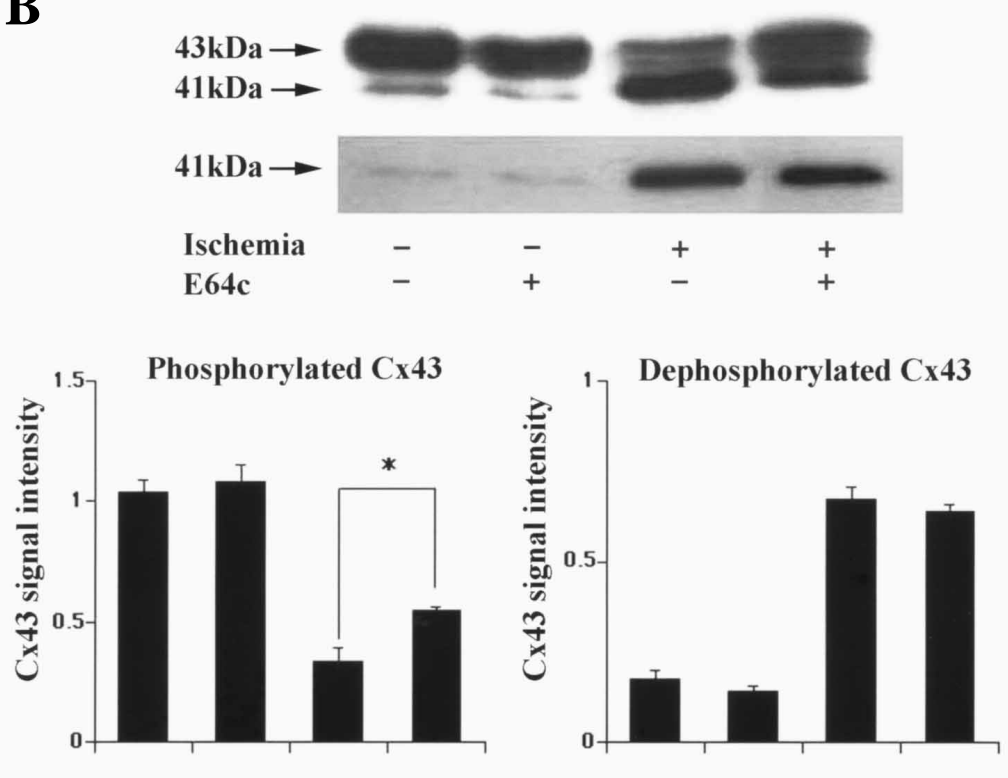

Ischemia E64c

Ischemia E64c

Figure 5. Representative immunoblots with the anti-Cx43 polyclonal antibody (upper) and monoclonal antibody (lower) showing the effects of PSI (A) and E64c (B) on phosphorylated Cx43 and dephosphorylated Cx43 levels at 1 hour of ischemia and in the sham. The relative densities of phosphorylated Cx43 and dephosphorylated $\mathrm{Cx} 43$ are quantified as ratios relative to the mean densities of the sham without inhibitors $(* P<0.05$ versus ischemia without inhibitor). 
A
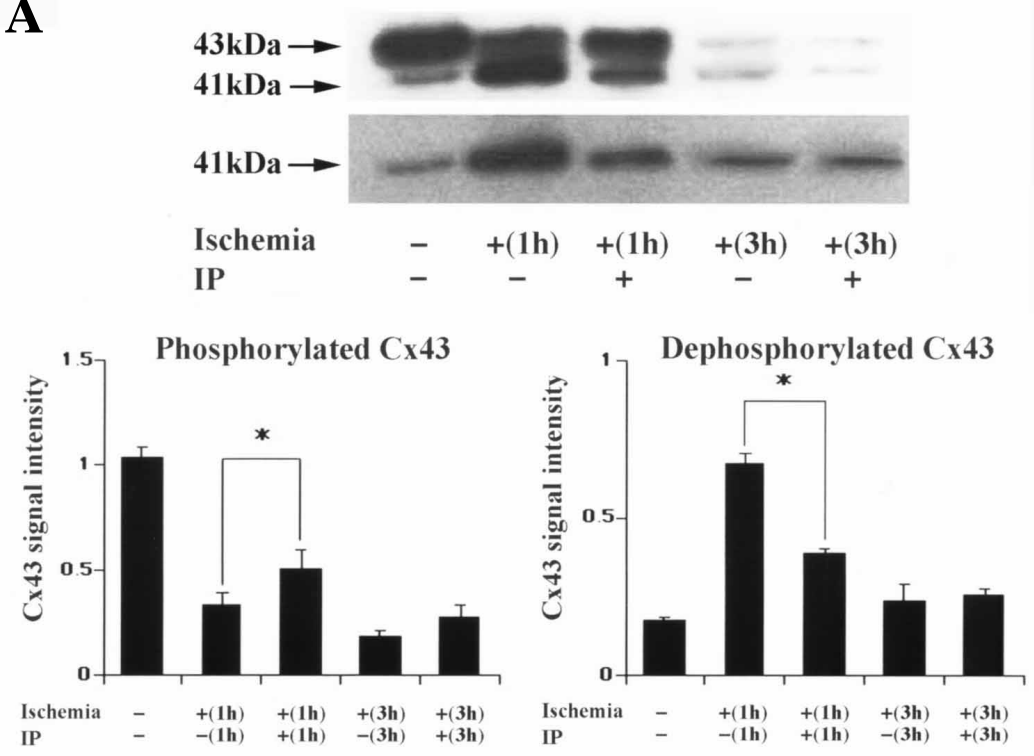

B

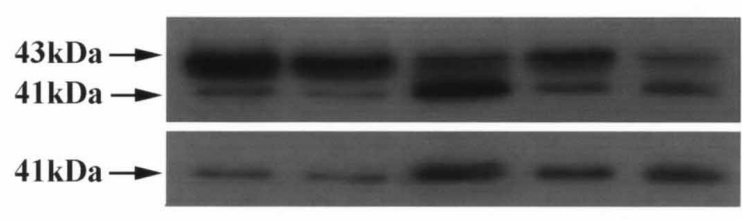

$\begin{array}{llllll}\text { Ischemia } & - & - & + & + & + \\ \text { IP } & - & - & - & + & + \\ \text { Che } & - & + & - & - & +\end{array}$
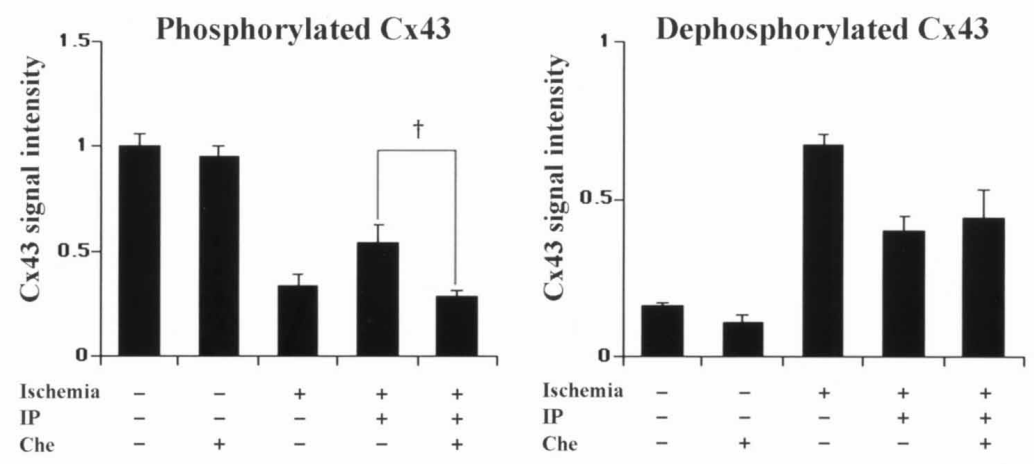

Figure 6. Representative immunoblots with the anti-Cx43 polyclonal antibody (upper) and the monoclonal antibody (lower) showing the effects of ischemic preconditioning (IP) (A) and the PKC inhibitor chelerythrine (Che) (B) on phosphorylated Cx43 and dephosphorylated Cx43 levels at 1 hour of ischemia. The relative densities of phosphorylated $\mathrm{Cx} 43$ and dephosphorylated $\mathrm{Cx} 43$ are quantified as ratios relative to the mean densities of the sham $\left(* P<0.05\right.$ versus ischemia without IP, ${ }^{\dagger} P<0.05$ versus IP without chelerythrine). 

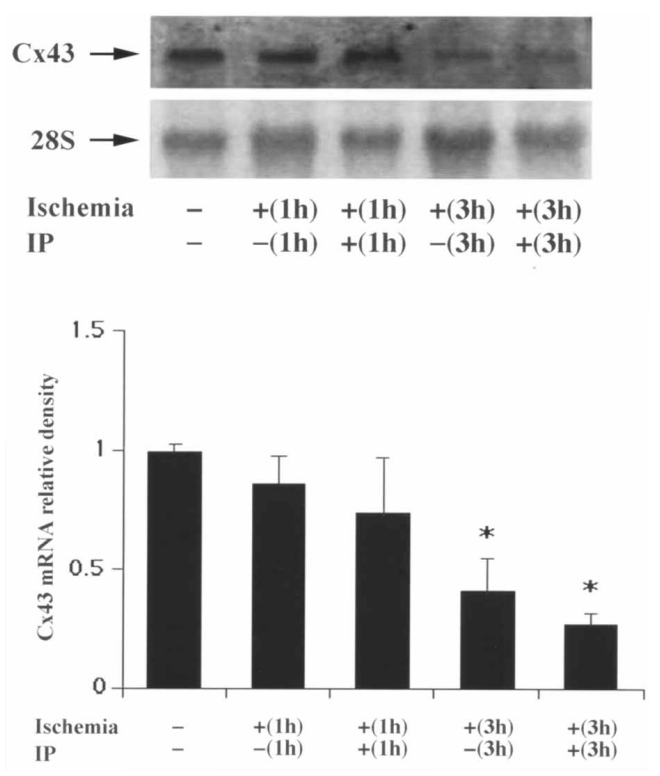

Figure 7. Representative Northern blot showing Cx43 mRNA after 1 and 3 hours of ischemia/ \pm IP. The density of each $\mathrm{Cx} 43$ band was normalized with reference to that of the $28 \mathrm{~S}$ rRNA band for each sample and expressed as ratios relative to the mean density of the sham $(* P<0.05$ versus sham $)$.

E64c (Figure 5B), on Cx43 levels after ischemia for 1 hour. PSI and E64c both inhibited the $\mathrm{pCx} 43$ decrease to a similar extent, with no effect on $\mathrm{dCx} 43$. In the nonischemic heart, the inhibitors per se did not increase the $\mathrm{Cx} 43$ band intensity. Effects of ischemic preconditioning and PKC inhibition on Cx43 levels: As shown in Figure 6A, IP inhibited the decrease in $\mathrm{pCx} 43$ and increase in $\mathrm{dCx} 43$ at 1 hour, but not at 3 hours of ischemia. However, a selective PKC inhibitor chelerythrine inhibited the effect of IP on the $\mathrm{pCx} 43$ level but not that on $\mathrm{dCx} 43$ level at 1 hour of ischemia, as demonstrated in Figure 6B. Chelerythrine had no effect on Cx43 levels in normoxic heart. We used chelerythrine because of its selectivity and effectiveness from our previous studies in perfusion and permanent occlusion models in the rat heart. ${ }^{10,26)}$

Cx43 mRNA levels: As shown in Figure 7, Cx43 mRNA was decreased at 3 hours but not at 1 hour of ischemia, irrespective of IP, suggesting transcriptional down-regulation in the later phase of ischemia.

\section{DISCUSSION}

We demonstrated for the first time that proteolysis of $\mathrm{Cx} 43$ is enhanced, while transcription is suppressed during early myocardial ischemia in an in vivo 
permanent occlusion model of the rat. At 1 hour of ischemia, $\mathrm{dCx} 43$ was largely increased, which was inhibited by a calcineurin inhibitor. $\mathrm{pCx} 43$ was decreased by $15 \%$, which was inhibited either by a lysosomal inhibitor or proteasome inhibitor. IP inhibited these changes, though PKC inhibition suppressed only the change in $\mathrm{pCx} 43$. The down-regulation of $\mathrm{Cx} 43$ may cause reduced $\mathrm{Cx} 43 \mathrm{immu}-$ nostaining postischemia. ${ }^{7)}$

By 1 hour of ischemia, a large portion of $\mathrm{pCx} 43$ was dephosphorylated and a small portion of $\mathrm{Cx} 43$ was supposed to be degraded (Figures 1,2), which is consistent with a previous study showing that $\mathrm{Cx} 43$ is dephosphorylated in the perfused heart rendered ischemic for 40 minutes. ${ }^{6}$ ) This $\mathrm{Cx} 43$ dephosphorylation during 1 hour of ischemia, as shown by the conversion of $\mathrm{pCx} 43$ to $\mathrm{dCx} 43$, was clearly inhibited by CsA (Figure 4), indicating the involvement of calcineurin. However, CsA did not affect the $\mathrm{Cx} 43$ down-regulation at 1 hour of myocardial ischemia. Additionally, both $\mathrm{pCx} 43$ and $\mathrm{dCx} 43$ were reduced with the duration of ischemia from 1 to 3 hours, supporting degradation independent of dephosphorylation. On the basis of these findings, we believe that proteolysis rather than "epitope masking by dephosphorylaiton" causes the "reduced immunostaining for Cx43 in ischemic myocardium".,3) Additionally, these changes precede the development of myocardial injury as demonstrated by the negative myoglobin diffusion or reduced triphenyltetrazolium chloride staining (data not shown), in the same model in which these changes can be reproduced after ischemia-reperfusion or prolonged ischemia (data not shown).

From the results of inhibitor studies in normoxic perfused hearts and cardiomyocytes, it has been formerly proposed that lysosomal proteases preferentially degrade pCx43 and other internalized membrane proteins after endocytosis, whereas proteasomes degrade $\mathrm{dCx} 43$, miss-folded or oxidized proteins during transport through the endoplasmic reticulum/Golgi apparatus in cardiomyocytes. ${ }^{1,8)}$ However, we found that a lysosomal inhibitor and a proteasomal inhibitor suppressed the reduction in the $\mathrm{pCx} 43$ at 1 hour of ischemia, though to a much smaller extent than the increase in $\mathrm{dCx} 43$ (Figure 5). These findings also appear to contradict the causality between dephosphorylation and proteolysis as well as the preference of the two protease systems in in vivo myocardial ischemia. The finding that E64c and PSI inhibited pCx43 reduction to similar extents suggests that both lysosomes and proteasomes are sequentially or simultaneously involved in $\mathrm{Cx} 43$ degradation in myocardial ischemia.

Immunohistochemical study showed that $\mathrm{dCx} 43$ label (by pAb) in the ID became distinct at 1 hour of ischemia, whereas $\mathrm{pCx} 43$ and $\mathrm{dCx} 43$ labels (by $\mathrm{mAb}$ ) became diffusely distributed to the intracellular spaces thereafter (Figure 3 ). Since lysosomal and proteasomal proteases are distributed to the intracellular spaces but not to the ID, these findings suggest that $\mathrm{Cx} 43$ undergoes proteolysis 
in situ beneath the ID or after endocytosis. The protease inhibitors were ineffective in the reductions in pCx43 and $\mathrm{dCx} 43$ at 3 hours of ischemia. This is not surprising, as no more inhibitor can reach the ischemic area after permanent coronary occlusion. On the other hand, consistent with the enhanced immunolabeling with time of ischemia throughout the myocardium (Figure 3), the Cx43 immunoreactivity in the $\mathrm{S}$ fraction on dot blots was increased with ischemia duration (Figure 2D). These data suggest that, as a result of rapid and efficient degradation during myocardial ischemia, the $\mathrm{Cx} 43$ epitope accumulates in the cytosol. Alternatively, given that the anti-pCx43 antibody is raised against its $\mathrm{C}$-terminal peptides, the immuno-labeling data could be explained if the $\mathrm{C}$-terminal region of Cx43 facing the cytoplasm was to be partially cleaved during early myocardial ischemia. Collectively, our data have clarified for the first time that myocardial ischemia reduced $\mathrm{Cx} 43$ levels by enhanced proteolysis. In addition to the enhanced degradation, the $\mathrm{Cx} 43$ level is supposed to be reduced by transcriptional down-regulation at 3 hours of ischemia because the Cx43 mRNA level was greatly reduced at 3 hours of ischemia (Figure 7).

IP inhibited the decrease in $\mathrm{pCx} 43$ level and increase in $\mathrm{dCx} 43$ at 1 hour postischemia, but only the change in $\mathrm{pCx} 43$ was suppressed by the PKC inhibitor (Figure 6). This observation suggests that PKC mediates the effect of IP on proteolysis rather than that on dephosphorylation as reported in previous immunohistochemical studies. ${ }^{15,16)}$ However, we were unable to discriminate between the two mechanisms due to the limitations of study in an in vivo model. To address the latter issue, we have begun studies using molecular and cell biological approaches.

It is reported that IP attenuates cardiac arrhythmias and infarct development, ${ }^{9,10)}$ while previous studies on $\mathrm{Cx} 43$ knock-out mice support the link between $\mathrm{Cx} 43$ down-regulation and arrhythmias. ${ }^{5)}$ It was also shown that a gap junction blocker, heptanol, attenuates the protective effect of IP. ${ }^{25}$ These data suggest that IP afforded protection against myocardial ischemia through the propagation of survival signals through $\mathrm{Cx} 43$-based GJ. The pathophysiological implications need to be examined in further molecular and cell biological studies.

In conclusion, during early ischemia ( 1 hour), Cx43 undergoes dephosphorylation and to a smaller extent proteolysis. During the later phase of ischemia ( $\sim 3$ hours), transcription is suppressed and proteolysis is enhanced.

\section{REFERENCES}

1. Saffitz JE, Laing JG, Yamada KA. Connexin expression and turnover; implications for cardiac excitability. Circ Res 2000; 86: 723-8. (Review) 
2. Huang XD, Sandusky GE, Zipes DP. Heterogeneous loss of connexin43 protein in ischemic dog hearts. J Cardiovasc Electrophysiol 1999; 10: 79-91.

3. Matsushita T, Takamatsu T. Ischaemia-induced temporal expression of connexin 43 in rat heart. Virchows Arch 1997; 431: 453-8.

4. Peters NS, Coromilas J, Severs NJ, Wit AL. Disturbed connexin43 gap junction distribution correlates with the location of reentrant circuits in the epicardial border zone of healing canine infarcts that cause ventricular tachycardia. Circulation 1997; 95: 988-96.

5. Lerner DL, Yamada KA, Schuessler RB, Saffitz JE. Accelerated onset and increased incidence of ventricular arrhythmias induced by ischemia in Cx43-deficient mice. Circulation 2000; 101: 547-52.

6. Beardslee MA, Lerner DL, Tadros PN, et al. Dephosphorylation and intracellular redistribution of ventricular connexin43 during electrical uncoupling induced by ischemia. Circ Res 2000; 87: 656-62.

7. Li WE, Nagy JI. Connexin 43 phosphorylation state and intercellular communication in cultured astrocytes following hypoxia and protein phosphatase inhibition. Eur J Neurosci 2000; 12: 2644-50.

8. Beardslee MA, Laing JG, Beyer EC, Saffitz JE. Rapid turnover of connexin43 in the adult rat heart. Circ Res 1998; 83: 629-35.

9. Parratt J, Vegh A. Pronounced antiarrhythmic effects of ischemic preconditioning. Cardioscience 1994; 5: 9-18. (Review)

10. Kawata H, Yoshida K, Kawamoto A, et al. Ischemic preconditioning upregulates vascular endothelial growth factor mRNA expression and neovascularization via nuclear translocation of protein kinase $\mathrm{C}$ epsilon in the rat ischemic myocardium. Circ Res 2001; 88: 696-704.

11. Sasaki Y, Sakai R, Furuta S. Evaluation of repeated balloon inflation in angioplasty as a clinical model of ischemic preconditioning. Jpn Heart J 1995; 36: 719-28.

12. Hiraoka M. Pathophysiogical functions of ATP-sensitive $\mathrm{K}^{+}$channels in myocardial ischemia. Jpn Heart $\mathrm{J}$ 1997; 38: 297-315.

13. Schwanke U, Konietzka I, Duschin A, Li X, Schulz R, Heusch G. No ischemic preconditioning in heterozygous connexin43-deficient mice. Am J Physiol Heart Circ Physiol 2002; 283: H1740-2.

14. Miura T, Ohnuma Y, Kuno A, et al. Protective role of gap junctions in preconditioning against myocardial infarction. Am J Physiol Heart Circ Physiol 2004; 286: H214-21.

15. Schulz R, Gres P, Skyschally A, et al. Ischemic preconditioning preserves connexin 43 phosphorylation during sustained ischemia in pig hearts in vivo. FASEB J 2003; 17: 1355-7.

16. Jain SK, Schuessler RB, Saffitz JE. Mechanism of delayed electrical uncoupling induced by ischemic preconditioning. Circ Res 2003; 92: 1138-44.

17. Squadrito F, Altavilla D, Squadrito G, et al. Cyclosporin-A reduces leukocyte accumulation and protects against myocardial ischemia reperfusion injury in rats. Eur J Pharmacol 1999; 364: 159-68.

18. Ohshita T, Nikawa T, Towatari T, Katunuma N. Effects of selective inhibition of cathepsin B and general inhibition of cysteine proteinases on lysosomal proteolysis in rat liver in vivo and in vitro. Eur J Biochem 1992; 209: 223-31.

19. Takaoka M, Itoh M, Hayashi S, Kuro T, Matsumura Y. Proteasome participates in the pathogenesis of ischemic acute renal failure in rats. Eur J Pharmacol 1999; 384: 43-6.

20. Fukuda S, Harada K, Kunimatsu M, Sakabe T, Yoshida K. Postischemic reperfusion induces $\alpha$-fodrin proteolysis by m-calpain in the synaptosome and nucleus in rat brain. J Neurochem 1998; 70: 2526-32.

21. Gray EG, Whittaker VP. The isolation of nerve endings from brain: an electron-microscopic study of cell fragments derived by homogenization and centrifugation. J Anat 1962; 96: 79-88.

22. Shinohara K, Funabashi T, Mitsushima D, Kimura F. Effects of estrogen on the expression of connexin32 and connexin43 mRNAs in the suprachiasmatic nucleus of female rats. Neurosci Lett 2000; 286: 107-10.

23. Nagy JI, Li WE, Roy C, et al. Selective monoclonal antibody recognition and cellular localization of an unphosphorylated form of connexin43. Exp Cell Res 1997; 236: 127-36.

24. Yoshida K, Inui M, Harada K, et al. Reperfusion of rat heart after brief ischemia induces proteolysis of calspectin (nonerythroid spectrin or fodrin) by calpain. Circ Res 1995; 77: 603-10.

25. Li G, Whittaker P, Yao M, Kloner RA, Przyklenk K. The gap junction uncoupler heptanol abrogates infarct size reduction with preconditioning in mouse hearts. Cardiovasc Pathol 2002; 11: 158-65. 